\title{
Association of ALDH1 with Response to Radiotherapy and Its Impact on Survival in Patients with Advanced Stage of Head and Neck Squamous Cell Carcinoma (HNSCC)
}

\author{
Parul Dubey ${ }^{1}$, Narayan Kumar ${ }^{2}$, Rajeev Gupta ${ }^{1}$, Anupam Mishra ${ }^{3}$, Smrati \\ Bhadauria $^{2}$, Vijay Kumar ${ }^{4}$, Madanlal Brahma Bhatt ${ }^{1 *}$
}

\begin{abstract}
Background: The presence of cancer stem-like cells within tumor microenvironment distinctly governs response to chemo-radiotherapy. The ALDH1 (Aldehyde dehydrogenase 1) has emerged as a cancer stem cell (CSC) marker in various tumors. The aim of the study was to examine the expression of ALDH1 in HNSCC patients undergoing radiotherapy to evaluate its correlation with clinicopathological parameter, treatment response and survival. Methods: Expression of ALDH1 was evaluated by immunohistochemistry in 90 histopathologically confirmed HNSCC patients and 90 matched controls. The association between ALDH1 expression, clinicopathological parameters and treatment response was determined. Results: The immunohistochemistry results showed that ALDH1 was consistently expressed in all the HNSCC specimens although at different intensities. On the other hand, control specimens did not show similar expression of ALDH1. ALDH1 expression demonstrated statistically significant association with tumor size $(p<0.001)$, lymph node status $(\mathrm{p}<0.001)$, stage $(\mathrm{p}<0.001)$, grade $(\mathrm{p}<0.001)$ and treatment response $(\mathrm{p}<0.001)$. Multivariate ordinal logistic regression analysis indicated alcohol and ALDH1 as an independent predictor of responsiveness to radiotherapy in HNSCC patients. Multivariate Cox regression analysis indicated that lymph node status $(p=0.020)$, grade $(p=0.006)$ and recurrence $(\mathrm{p}=0.002)$ were potential independent predictors of overall survival. Conclusion: From previous studies, ALDH1 has been contemplated not only as a promising prognostic and diagnostic marker but also as a likely drug target. Our study gives new understanding regarding the association between ALDH1, cancer prognosis and radioresistance. Our findings suggest that ALDH1, lymph node status, grade and alcohol could be the viable targets for HNSCC and it also provides new prospects for radiotherapy sensitivity in HNSCC.
\end{abstract}

Keywords: Cancer stem cells - Immunohistochemistry - Aldehyde dehydrogenase 1 - Radiotherapy

Asian Pac J Cancer Prev, 23 (2), 419-427

\section{Introduction}

Head and neck squamous cell carcinoma (HNSCC) is one of the sixth most common malignancies worldwide (Shaw and Beasley 2016). It accounts for about 550,000 new cases and 380,000 deaths per year (Fitzmaurice et al., 2017). HNSCC is a heterogeneous group of various malignancies which involves oral cavity, pharynx and larynx. A 5-year survival study was conducted between 2006 to 2012 by the American Cancer Society indicated that patients with oral cavity and pharynx cancers have $64 \%$ of 5-year survival. However, patients with larynx cancer have $61 \%$ of 5 -year survival (Siegel et al., 2017). Due to failure in early diagnosis, HNSCC is still associated with low survival rates despite the advancement in the understanding and treatment of HNSCC. As a matter of fact, at an early stage only one third of patients were diagnosed with HNSCC (Patel and Shah 2005; McCullough et al., 2010; Economopoulou et al., 2019). Due to the onset of metastasis and development of resistance to therapies, mortality remains high (Mannelli and Gallo, 2012). Regardless of the advancement in the current treatment strategies, the prognosis of HNSCC remains poor due to late stage at diagnosis, high rates of primary site recurrence, and frequent metastases to locoregional lymph nodes (McCullough et al., 2010). Recently, cancer stem cells (CSCs) have been attributed for the poor treatment outcome and survival in many cancers including HNSCC.

Cancer stem cell (CSC) hypothesis states that a small subset of cells which are phenotypically different are found in most of the solid tumors. These cells have stem 
cell-like properties such as self renewal, proliferation and differentiation along with inherent chemo-radio resistance and increased clonogenic potential (Alison et al., 2012). Due to the self renewal capacity of CSCs, they have the ability to never-ending proliferation and excessive tumorigenicity. The stem-ness of CSCs may be indicated by these attributes (Alamgeer et al., 2013). Cancer stem cells (CSCs) were originally identified in hematologic cancers (Lapidot et al., 1994). However, according to various studies they have been found in various cancers including breast (Al-Hajj et al., 2003), brain (Singh et al., 2003), liver (Wu et al., 1999), colon (Ricci-Vitiani et al., 2007), lung (Kim et al., 2005), bladder (Chan et al., 2009) and head and neck (Prince et al., 2007). These cells possess three properties: self-renewal, differentiation and proliferation which are equivalent to the properties of normal stem cells (Weissman et al., 2001). Resistance potential of CSCs to radiation therapy and chemotherapy is one of the important aspects (Jordan et al., 2006). On this basis, it has been postulated that CSCs are one of the key determinants of treatment failure and tumor recurrence (Baumann et al., 2008). Therefore, identification of new diagnostic CSC markers could be important for such cancers.

CSCs can be identified by various putative surface markers that are expressed by them. Numerous independent studies have shown that among all the CSC markers expressed in HNSCC, the Aldehyde Dehydrogenase 1 (ALDH1) is the most consistently identified CSC marker. In HNSCC-derived CSCs, ALDH1 also plays a critical role in retaining the self renewal properties and tumorigenicity (Clay et al., 2010; Chen et al., 2011). Currently, ALDH super family comprises 19 known functional genes in 11 families which further contain 4 sub-families. It has been proclaimed that ALDH consists ofALDH1 family, ALDH2*2, ALDH3A1, ALDH4A1 and ALDH7A1 (Black et al., 2009). It has also been reported that ALDH1 family includes ALDH1A1, ALDH1A2 and ALDH1A3 sub-families (Li et al., 2014). ALDH1 is a cytosolic enzyme and its function is to convert retinaldehyde to retinoic acid (RA), which plays a critical role in cellular proliferation and differentiation (Singh et al., 2013). ALDH1 is the key factor for the maintenance and differentiation of stem cells (Clarke and Palle, 2016). Ma et al., (2015) had reported that there is an association between high ALDH1 expression and advanced tumor stage, tumor size and lymph node metastasis in breast cancer. Earlier studies have revealed definite prognostic values of ALDH1 isoforms in non-small cell lung cancer, breast cancer and gastric cancer (You et al., 2014; Wu et al., 2015; Shen et al., 2016).

Though, multiple studies have been conducted on HNSCC cell lines and cell lines derived from primary tumor but it's hard to maintain the physiology of tumor in vivo and ex vivo and to develop a tumor microenvironment or the niche which is required for a stem cell to grow. Furthermore, there have been studies which involve isoforms of ALDH1 in case of HNSCC as well as other malignancies but no studies have been conducted so far on patient samples with ALDH1 in HNSCC. In addition, Martin et al., (2016) had reported that in laryngeal carcinoma, patients with tumors expressing ALDH1 had better outcome in terms of chemo-radiotherapy and overall survival (OS). This implies as a contradictory to other studies which demonstrated that presence of ALDH1 leads to the resistance to chemo-radiotherapy and had poor OS. Therefore, the current study was designed to evaluate the predictive significance of ALDH1 in terms of radiotherapy and OS in advanced stage patients of HNSCC.

\section{Materials and Methods}

\section{Patients and Tissue Samples}

Total 90 histopathologically confirmed HNSCC patients and 90 age and gender matched controls attending dental procedures for dental implant or benign cyst were recruited for the evaluation of a CSC marker "ALDH1" by immunohistochemistry. All HNSCC nonmetastatic patients from 2015 to 2017 , recommended for radiotherapy were enrolled in the study. Written informed consent was obtained from all the patients. All patients underwent biopsy and samples from formalin-fixed paraffin-embedded tissues were obtained. The study was approved by Institutional Ethics Committee. Patients more than 18 years of age and normal hematological, renal and liver function were included. Patients with history of prior radiotherapy, chemotherapy and surgery were excluded.

\section{Treatment and Response Assessment}

All patients were subjected to standard treatment of radiotherapy by using linear accelerator. A dose of $70 \mathrm{~Gy} / 35$ fractions, $5 \mathrm{Fc} /$ week of radiation was delivered in 7 weeks in two phases: Phase I- 46Gy/23\# to primary tumor and whole neck and phase II- 24Gy/12\# to tumor and neck sparing the cord. After one month of completion of radiotherapy treatment, the tumor response was assessed by clinical examination and radiological investigation, for instance, CT-scan. The treatment response, viz., complete response $(\mathrm{CR})$, partial response $(\mathrm{PR})$ and no response (NR) [stable disease (SD) + progressive disease (PD)] were based on the guidelines defined by a new set of tumor response criteria, i.e., Response Evaluation Criteria in Solid Tumors (RECIST) (Eisenhauer et al., 2009). To find out the role of CSCs as a predictive biomarker of radiotherapy response, ALDH1 expression level was correlated with radiotherapy response after treatment and evaluation.

\section{Immunohistochemistry}

CSC marker ALDH1 was detected by immunohistochemistry which was performed on formalin-fixed, paraffin-embedded tissue sections $(5 \mu \mathrm{m})$. Blocking of peroxidases was done on deparafinized, rehydrated slides by using $0.1 \%$ hydrogen peroxide after heat-induced epitope retrieval (HIER) with citrate buffer ( $\mathrm{pH}$ 6.0). Sections were then incubated overnight with primary antibody (ALDH1 (H-8): sc-166362 Santa Cruz Biotechnology, California, USA), at 1:50 dilution. Thereafter, samples were incubated with HRP-conjugated goat anti-mouseIgG for $1 \mathrm{hr}$ at 1:200 dilutions. Detection was performed with DAB peroxidase substrate kit (Thermo FisherScientific, Waltham, MA, 
USA), followed by counterstaining with hematoxylin (ThermoFischerScientifc, USA). Sections were mounted in DPX (Sigma) and analyzed at 40X magnification using Leica DCF450C bright field/fluorescence microscope.

\section{Scoring for ALDH1 Expression}

Evaluation of ALDH1 immunostaining was done on whole tissue section of both control samples and HNSCC patient samples. Ten different fields per section were viewed and cell percentage positivity for ALDH1 staining was evaluated as, low for $<25 \%$ staining and high for $\geq 25 \%$ staining (Collina et al., 2015). No staining was considered as negative staining.

\section{Statistical Analysis}

Continuous data were summarized as Mean $\pm \mathrm{SE}$ while discrete (categorical) in number (n) and percentage $(\%)$. Continuous groups were compared by independent Student's t test. Categorical groups were compared by chi-square $\left(\chi^{2}\right)$ test. Univariate and multivariate ordinal logistic regression analysis was done to assess independent predictor(s) of responsiveness to radiotherapy. Survival analysis was done using Kaplan-Meier method and difference between groups was done by Log-rank test. A two-tailed $(\alpha=2) \mathrm{p}<0.05$ was considered statistically significant.

\section{Results}

\section{Basic Characteristics of HNSCC Patients}

The demographic parameters, clinico-pathological parameters and treatment response of HNSCC patients, $\mathrm{n}=90$ are summarized in Table 1 . The age of patients ranged from 22-74 yrs with mean age $( \pm$ SE) $46.64 \pm 1.19$ yrs and median age $45 \mathrm{yrs}$. Majority of the patients were of age $\leq 45$ yrs $(54.4 \%)$ and larger section were males $(86.7 \%)$. Out of 90 patients, $69(76.7 \%)$ patients consumed tobacco and $37(41.1 \%)$ consumed alcohol.

In most of the patients the primary tumor site was buccal mucosa $20(22.2 \%)$. Further, the patients were mostly T4 tumor size (45.5\%), lymph node N1 (47.8\%), stage IV $(61.1 \%)$ and moderately differentiated (MD) grade $(44.5 \%)$.

Of total number of patients, $26(28.9 \%)$ had complete response (CR), 16 (17.8\%) had partial response (PR) and $48(53.3 \%)$ had no response (NR). Thus, the overall response $(\mathrm{CR}+\mathrm{PR})$ was $46.7 \%$.

\section{Assessment of Molecular Marker Expression}

The expression of molecular marker ALDH1 by immunohistochemistry is shown in Figure 1 (A). All the 90 patients positively expressed the marker ALDH1. Out of which, $20(22.2 \%)$ had low expression and $70(77.8 \%)$ had high expression. In contrast, in controls (patients attending dental procedures for dental implant or benign cyst) the expression of ALDH1 biomarker was negative, i.e., no staining and it is shown in Figure 1 (B).

Association of Treatment Response with Demographic, Clinico-pathological Parameters and Biomarker Expression
The association of treatment response with various variables (demographic and clinico-pathological parameters) is established. On correlating, the treatment response showed significant and direct (positive)

Table 1. Demographic, Clinico-Pathological Characteristics and Treatment Response of HNSCC Patients

\begin{tabular}{|c|c|}
\hline Characteristics & Cases $(n=90)(\%)$ \\
\hline \multicolumn{2}{|l|}{ Age (yrs) } \\
\hline$\leq 45$ & $49(54.4)$ \\
\hline$>45$ & $41(45.6)$ \\
\hline \multicolumn{2}{|l|}{ Gender: } \\
\hline Female & $12(13.3)$ \\
\hline Male & $78(86.7)$ \\
\hline \multicolumn{2}{|l|}{ Tobacco } \\
\hline No & $21(23.3)$ \\
\hline Yes & $69(76.7)$ \\
\hline \multicolumn{2}{|l|}{ Alcohol } \\
\hline No & $53(58.9)$ \\
\hline Yes & $37(41.1)$ \\
\hline \multicolumn{2}{|l|}{ Tumor Site } \\
\hline Buccal Muosa & $20(22.2)$ \\
\hline Border of Tongue & $18(20.0)$ \\
\hline Base of Tongue & $10(11.1)$ \\
\hline Others\# & $42(46.7)$ \\
\hline \multicolumn{2}{|l|}{ Tumor Size } \\
\hline $\mathrm{T} 1$ & $6(6.7)$ \\
\hline $\mathrm{T} 2$ & $16(17.8)$ \\
\hline T3 & $27(30.0)$ \\
\hline $\mathrm{T} 4$ & $41(45.5)$ \\
\hline \multicolumn{2}{|l|}{ Lymph Node Status } \\
\hline No & $6(6.7)$ \\
\hline N1 & $43(47.8)$ \\
\hline $\mathrm{N} 2$ & $41(45.5)$ \\
\hline \multicolumn{2}{|l|}{ Stage } \\
\hline III & $35(38.9)$ \\
\hline IV & $55(61.1)$ \\
\hline \multicolumn{2}{|l|}{ Grade } \\
\hline WD & $31(34.4)$ \\
\hline $\mathrm{MD}$ & $40(44.5)$ \\
\hline PD & $19(21.1)$ \\
\hline \multicolumn{2}{|l|}{ Response } \\
\hline $\mathrm{CR}$ & $26(28.9)$ \\
\hline PR & $16(17.8)$ \\
\hline NR & $48(53.3)$ \\
\hline
\end{tabular}

$\mathrm{T} 1, \leq 2 \mathrm{~cm}$ in greatest dimension; T2, $2-4 \mathrm{~cm}$ or affects 2 areas within a specific site; T3, $>4 \mathrm{~cm}$ or affects 3 areas within a specific site; T4, invades specific structures (4a is moderately advanced local disease and $4 \mathrm{~b}$ is very advanced local disease); N0, No lymph node metastasis; $\mathrm{N} 1$, One node $\leq 3 \mathrm{~cm}$; N2, Node between 3 and $6 \mathrm{~cm}$ or multiple nodes; WD, Well Differentiated; MD, Moderately Differentiated; PD, Poorly Differentiated; CR, Complete Response or Complete Responder; PR, Partial Response or Partial Responder; NR, No Response or No Responder; \#, Oral Cavity; Larynx, Laryngopharynx, Nasopharynx 
A

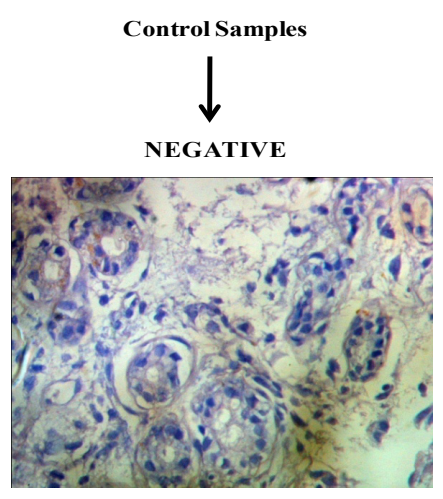

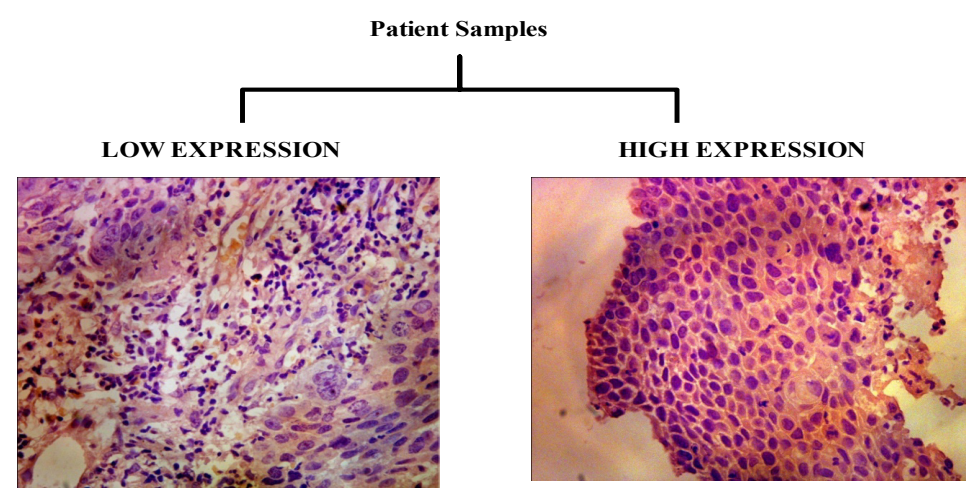

B
ALDH1 Expression
Control
$(\mathrm{n}=90)(\%)$

Low $(<25 \%) \quad$ Negative $\quad 20(22.2)$
$\operatorname{High}(\geq 25 \%)$

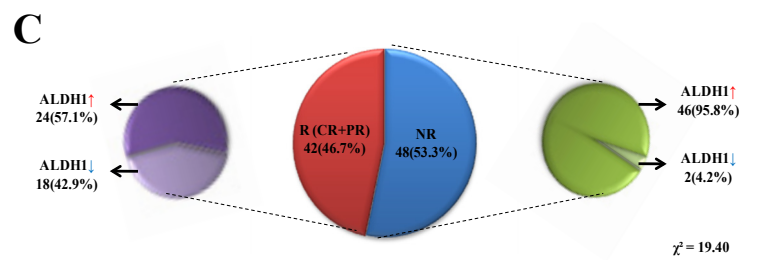

$\chi^{2}=19.40$
$\mathbf{p}<0.001 * * *$

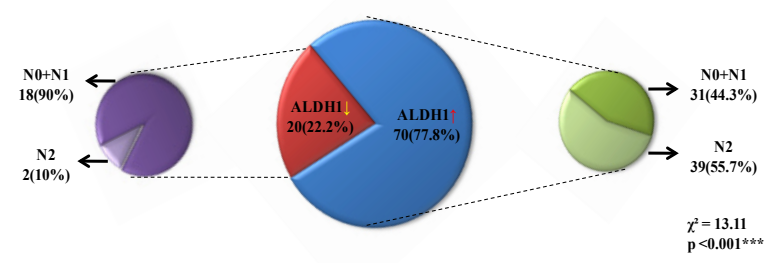

$70(77.8)$

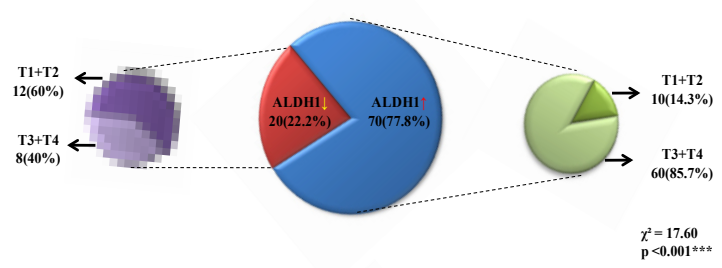

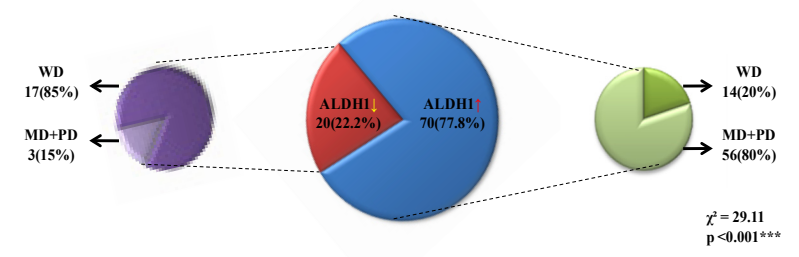

Figure 1. Immunohistochemistry Detection of ALDH1 in HNSCC Specimens and Pie Chart Depicting Association between Various Variables Using Chi-square Test. A, Micrograph showing negative staining from control samples and from patient samples showing low and high expression of positive stained nuclei; B, Frequency distribution of ALDH1 expression in control and HNSCC patients; C, Showing significant association between treatment response and ALDH1 expression $(\mathrm{p}<0.001)$; Showing significant association between ALDH1 expression and tumor size $(\mathrm{p}<0.001)$, lymph node status $(\mathrm{p}<0.001)$, stage $(\mathrm{p}<0.001)$ and grade $(\mathrm{p}<0.001)$.

association with tumor size $\left(\chi^{2}=30.36, \mathrm{p}<0.001\right)$, lymph node status $\left(\chi^{2}=15.19, \mathrm{p}=0.004\right)$, stage $\left(\chi^{2}=32.19\right.$, $\mathrm{p}<0.001)$ and grade $\left(\chi^{2}=21.12, \mathrm{p}<0.001\right)$. The significant correlation indicates that these variables may be associated with responsiveness or serve as the predictors of responsiveness.

The association of treatment response with biomarker expression is shown in Figure 1 (C). On correlating, the treatment response showed significant association with biomarker expression $\left(\chi^{2}=19.40, p<0.001\right)$.

Since, tumor size, lymph node status, stage, grade and biomarker expression showed the significant association with the treatment response, we next evaluated the association of biomarker expression with the above mentioned variables which is summarized in Figure 1 (C).

On correlating, the tumor size $\left(\chi^{2}=17.60, \mathrm{p}<0.001\right)$, 
Table 2. Identification of Predictor of Responsiveness to Radiotherapy in HNSCC Patients Using Ordinal Logistic Regression Analysis ( $\mathrm{n}=90)$

\begin{tabular}{|c|c|c|c|c|}
\hline Predictor & $\begin{array}{c}\text { Univariate } \\
\text { OR }(95 \% \text { CI })\end{array}$ & $\begin{array}{l}\text { Column1 } \\
\text { p value }\end{array}$ & $\begin{array}{l}\text { Multivariate } \\
\text { OR }(95 \% \mathrm{CI})\end{array}$ & $\begin{array}{c}\text { Column } 2 \\
\text { p value }\end{array}$ \\
\hline \multicolumn{5}{|l|}{ Age (yrs) } \\
\hline$\leq 45$ & Ref & & & \\
\hline$>45$ & $0.71(0.33-1.56)$ & 0.399 & & \\
\hline \multicolumn{5}{|l|}{ Gender } \\
\hline Female & Ref & & & \\
\hline Male & $1.04(0.33-3.27)$ & 0.945 & & \\
\hline \multicolumn{5}{|l|}{ Tobacco } \\
\hline No & Ref & & & \\
\hline Yes & $0.74(0.30-1.85)$ & 0.52 & & \\
\hline \multicolumn{5}{|l|}{ Alcohol } \\
\hline No & Ref & & Ref & \\
\hline Yes & $0.28(0.12-0.64)$ & $0.003^{*}$ & $0.29(0.11-0.75)$ & $0.011^{*}$ \\
\hline \multicolumn{5}{|l|}{ Tumor Size } \\
\hline $\mathrm{T} 1+\mathrm{T} 2$ & Ref & & Ref & \\
\hline $\mathrm{T} 3+\mathrm{T} 4$ & $0.33(0.13-0.83)$ & $0.019^{*}$ & $2.14(0.52-8.81)$ & 0.29 \\
\hline \multicolumn{5}{|c|}{ Lymph Node Status } \\
\hline N0 + N1 & Ref & & Ref & \\
\hline $\mathrm{N} 2$ & $0.30(0.13-0.69)$ & $0.005^{*}$ & $1.06(0.34-3.31)$ & 0.922 \\
\hline \multicolumn{5}{|l|}{ Stage } \\
\hline III & Ref & & Ref & \\
\hline IV & $0.14(0.06-0.34)$ & $<0.001 * * *$ & $0.31(0.07-1.28)$ & 0.104 \\
\hline \multicolumn{5}{|l|}{ Grade } \\
\hline WD & Ref & & Ref & \\
\hline $\mathrm{MD}+\mathrm{PD}$ & $0.30(0.13-0.71)$ & $0.006^{*}$ & $1.08(0.33-3.57)$ & 0.898 \\
\hline \multicolumn{5}{|l|}{ ALDH1 } \\
\hline Low & Ref & & Ref & \\
\hline High & $0.06(0.02-0.18)$ & $<0.001 * * *$ & $0.06(0.01-0.30)$ & $0.001 * *$ \\
\hline
\end{tabular}

WD, Well Differentiated; MD, Moderately Differentiated; PD, Poorly Differentiated; CR, Complete Response or Complete Responder; PR, Partial Response or Partial Responder; NR, No Response or Non Responder; OR, Odds Ratio; CI, Confidence Interval; Ref, Reference Category. The mentioned odd ratio is evaluated against reference category; ${ }^{*}, \mathrm{p}<0.05 ; * *, \mathrm{p}<0.01 ; * * *, \mathrm{p}<0.001$.

lymph node status $\left(\chi^{2}=13.11, \mathrm{p}<0.001\right)$, stage $\left(\chi^{2}=28.27\right.$, $\mathrm{p}<0.001)$ and grade $\left(\chi^{2}=29.11, \mathrm{p}<0.001\right)$ showed significant association with biomarker expression.

\section{Predictors of Radiotherapy Response}

To find out the predictor (s) of response (CR, PR and $\mathrm{NR})$ to radiotherapy in HNSCC patients, the patients demographic characteristics (age, gender, tobacco and alcohol), clinico-pathological findings (tumor size, lymph node, stage and grade), and molecular biomarker (ALDH1) expression was examined against responsiveness. Univariate and multivariate ordinal logistic regression analysis was used.

Response to radiotherapy was considered as the dependent variable while demographic, clinicopathological and biomarker expression as independent variable and is summarized in Table 2.

Univariate ordinal logistic regression showed significant $(\mathrm{p}<0.05$ or $\mathrm{p}<0.01$ or $\mathrm{p}<0.001)$ association of alcohol, tumor size, lymph node status, stage, grade and ALDH1 expression with responsiveness suggesting these being potential predictors of responsiveness to radiotherapy in HNSCC patients. Moreover, subjecting significant predictors to multivariate analysis revealed that alcohol and ALDH1 expression are significantly $(\mathrm{p}<0.05$ or $\mathrm{p}<0.01$ or $\mathrm{p}<0.001)$ associated with responsiveness. This indicates that alcohol and ALDH1 being the potential significant and independent predictor of responsiveness to radiotherapy in HNSCC patients.

Overall Survival of HNSCC Patients and its Association with Clinico-pathological Characteristics, Biomarker Expression and Treatment Response

After treatment, the HNSCC patients were followed up for 2 years. During the period, $20(22.2 \%)$ patients died due to disease. However, 53 (58.9\%) patients were alive and 17 (18.9\%) lost to follow up (LOF or censored) accounting together total 70 alive (Alive + LOF). Thus the incidence of overall survival was $77.8 \%$. Further, during the assessment period 41 patients showed recurrence 
A

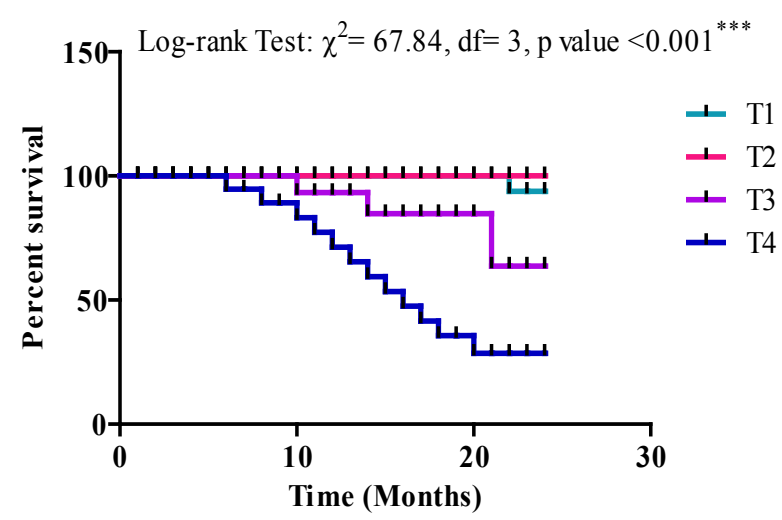

C

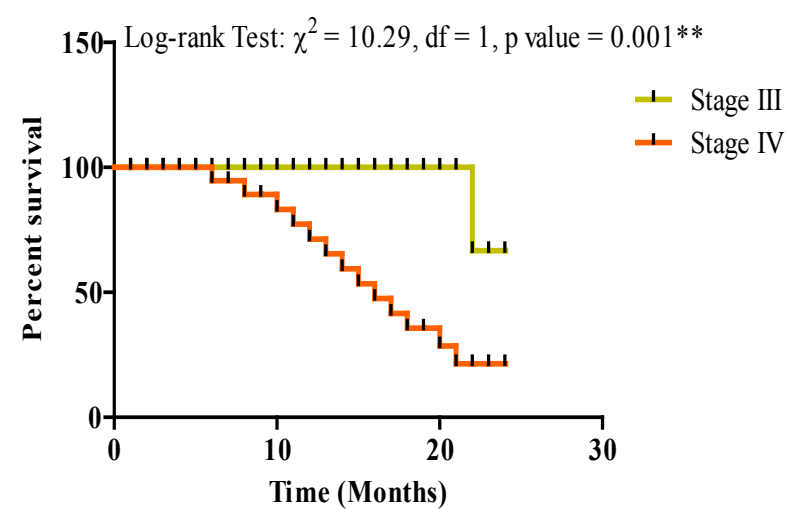

$\mathbf{E}$

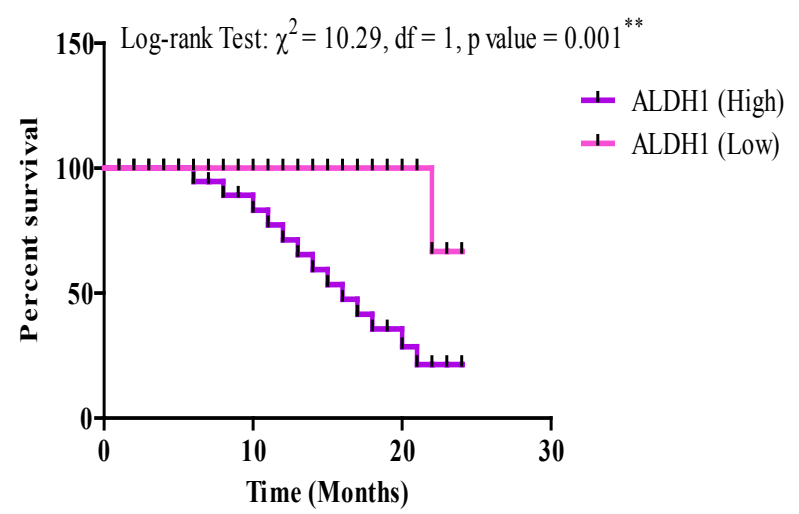

\section{B}

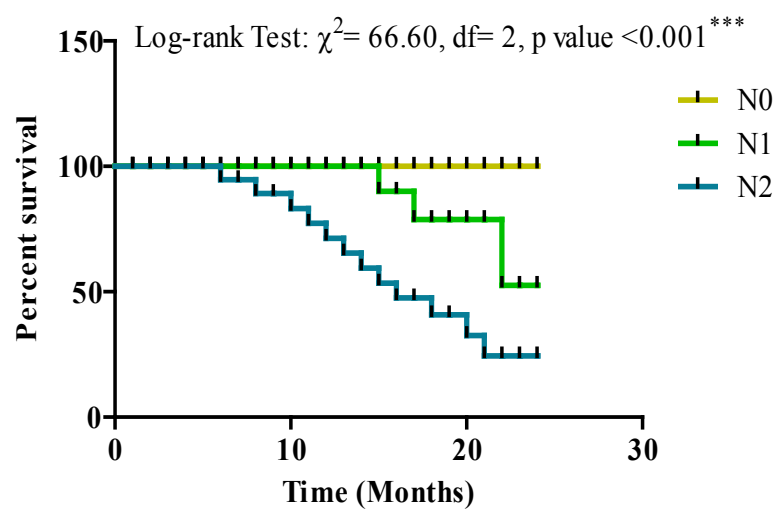

D

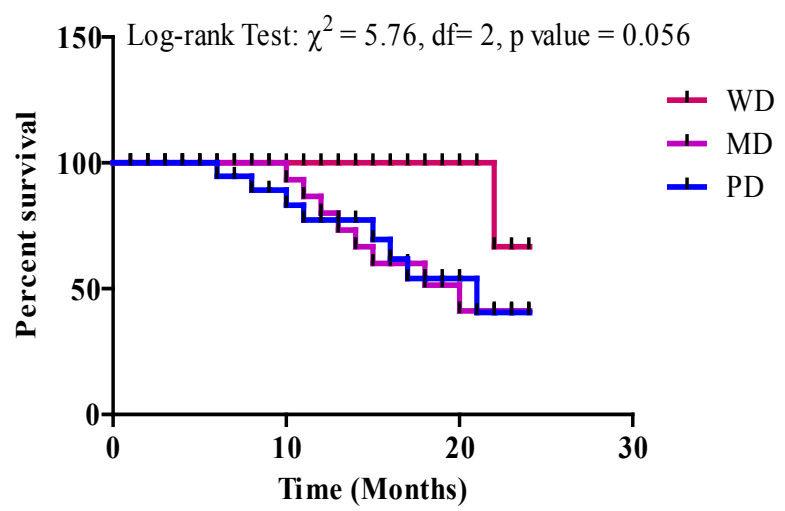

$\mathbf{F}$

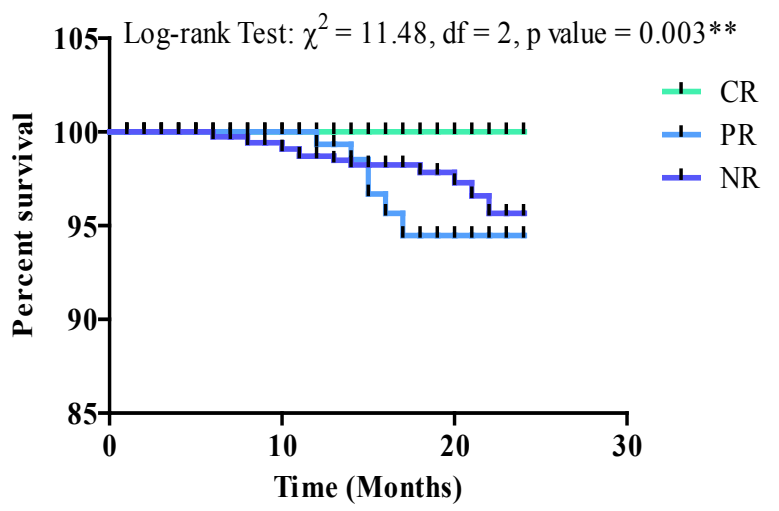

Figure 2. Two Year Overall Survival of HNSCC Patients Using Kaplan-Meier Method. A, Overall survival showing significant association with tumor size; B, Overall survival showing significant association with lymph node status; C, Overall survival showing significant association with stage; D, Overall survival showing no significant association with grade; E, Overall survival showing significant association with ALDH1 expression; F, Overall survival showing significant association with treatment response.

\section{$(45.6 \%)$}

The 2 year overall survival of HNSCC patients with clinico-pathological parameters, biomarker expression and treatment response was analyzed by using Kaplan-Meier method and the difference between groups was done using Log rank (Mantel-Cox) test which is summarized graphically in Figure 2. Our analysis using Log rank (Mantel-Cox) test showed significant ( $\mathrm{p}<0.05$ or $\mathrm{p}<0.01$ or $\mathrm{p}<0.001)$ association of all clinico-pathological characteristics except grade with overall survival. The Log rank test showed that as tumor size $\left(\chi^{2}=67.84, \mathrm{p}<0.001\right)$, lymph node $\left(\chi^{2}=66.60, \mathrm{p}<0.001\right)$ and stage $\left(\chi^{2}=10.29\right.$, $\mathrm{p}=0.001)$ increases the mean survival decreases. Further, the Log rank test showed that the mean survival also decreases significantly in patients with high expression of ALDH1 $\left(\chi^{2}=10.29, \mathrm{p}=0.001\right)$ as compared to respective low expression.

Moreover, on analyzing using Log rank test it was observed that treatment response (CR, PR and NR) was also significantly associated with the overall survival $\left(\chi^{2}=\right.$ 
DOI:10.31557/APJCP.2022.23.2.419

ALDH1 Role in Therapy Response and Survival

Table 3. Time Dependent Independent Predictors of Survival Using Univariate and Multivariate Cox Regression Analysis $(\mathrm{n}=90)$

\begin{tabular}{|c|c|c|c|c|}
\hline Predictor & $\begin{array}{c}\text { Univariate } \\
\text { OR }(95 \% \mathrm{CI})\end{array}$ & $\begin{array}{l}\text { Column } 1 \\
\text { p value }\end{array}$ & $\begin{array}{l}\text { Multivariate } \\
\text { OR }(95 \% \text { CI })\end{array}$ & $\begin{array}{c}\text { Column } 2 \\
\text { p value }\end{array}$ \\
\hline \multicolumn{5}{|l|}{ Age (yrs) } \\
\hline$\leq 45$ & Ref & & Ref & \\
\hline$>45$ & $0.91(0.60-1.38)$ & 0.667 & $0.85(0.51-1.41)$ & 0.518 \\
\hline \multicolumn{5}{|l|}{ Gender } \\
\hline Female & Ref & & Ref & \\
\hline Male & $1.13(0.62-2.08)$ & 0.69 & $1.11(0.55-2.23)$ & 0.777 \\
\hline \multicolumn{5}{|l|}{ Tobacco } \\
\hline No & Ref & & Ref & \\
\hline Yes & $0.95(0.59-1.56)$ & 0.851 & $0.78(0.45-1.34)$ & 0.365 \\
\hline \multicolumn{5}{|l|}{ Alcohol } \\
\hline No & Ref & & Ref & \\
\hline Yes & $1.41(0.93-2.15)$ & 0.108 & $1.40(0.86-2.29)$ & 0.176 \\
\hline \multicolumn{5}{|l|}{ Tumor Size } \\
\hline $\mathrm{T} 1+\mathrm{T} 2$ & Ref & & Ref & \\
\hline $\mathrm{T} 3+\mathrm{T} 4$ & $1.37(0.84-2.21)$ & 0.205 & $1.59(0.80-3.15)$ & 0.185 \\
\hline \multicolumn{5}{|c|}{ Lymph Node Status } \\
\hline N0 & Ref & & Ref & \\
\hline $\mathrm{N} 1+\mathrm{N} 2$ & $1.58(0.69-3.62)$ & 0.28 & $3.09(1.19-7.96)$ & $0.020^{*}$ \\
\hline \multicolumn{5}{|l|}{ Stage } \\
\hline III & Ref & & Ref & \\
\hline IV & $1.48(0.97-2.27)$ & 0.069 & $1.20(0.65-2.24)$ & 0.564 \\
\hline \multicolumn{5}{|l|}{ Grade } \\
\hline WD & Ref & & Ref & \\
\hline $\mathrm{MD}+\mathrm{PD}$ & $1.98(1.26-3.12)$ & $0.003^{*}$ & $2.25(1.27-4.00)$ & $0.006^{*}$ \\
\hline \multicolumn{5}{|l|}{ ALDH1 } \\
\hline Low & Ref & & Ref & \\
\hline High & $1.43(0.87-2.35)$ & 0.158 & $0.91(0.43-1.94)$ & 0.81 \\
\hline \multicolumn{5}{|l|}{ Response } \\
\hline $\mathrm{CR}+\mathrm{PR}$ & Ref & & Ref & \\
\hline NR & $1.43(0.94-2.16)$ & 0.093 & $1.33(0.80-2.20)$ & 0.271 \\
\hline \multicolumn{5}{|l|}{ Recurrence } \\
\hline No & Ref & & Ref & \\
\hline Yes & $0.93(0.61-1.41)$ & 0.723 & $0.41(0.24-0.72)$ & $0.002 *$ \\
\hline
\end{tabular}

WD, Well Differentiated; MD, Moderately Differentiated; PD, Poorly Differentiated; CR, Complete Response or Complete Responder; PR, Partial Response or Partial Responder; NR, No Response or Non Responder; OR, Odds Ratio; CI, Confidence Interval; Ref, Reference Category. The mentioned odd ratio is evaluated against reference category; * $\mathrm{p}<0.05$

$11.48, \mathrm{p}=0.003)$.

Independent predictors of survival

Lastly, to find out the independent predictors of overall survival (death and live + LOF), univariate and multivariate Cox regression analysis is done between different predictor variables (demographic, clinicopathological, biomarker expression, treatment response and recurrence) and survival which is summarized in Table 3.

In univariate analysis, no significant association was found between proposed markers with the survival. However, tumor grade was found to be exhibiting significant association $(\mathrm{p}<0.05, \mathrm{p}<0.01)$ with survival.
On the contrary, in multivariate analysis, lymph node status, grade and recurrence was found to be independently associated with survival. Thus, lymph node, grade and recurrence could serve as significant and independent predictors of survival after adjusting the variables.

\section{Discussion}

In recent years, several studies have been proposed that ALDH1 is associated with worse prognosis in various types of cancer and it could be the independent factor for poor OS (Alamgeer et al., 2013). Multiple studies have demonstrated that ALDH1 is expressed in almost all types 
of cancer tissues but to different extents (Liu et al., 2014; Zhou and Sun, 2014; Singh et al., 2015). A meta-analysis study on colorectal cancer showed that as an independent prognostic factor, the over expression of ALDH1 was found to be significantly associated with OS and diseasefree survival (DFS) rates, T stage, lymph node status and tumor differentiation. As a CSC marker, ALDH1 enhances the cell proliferation and invasion capacity of a tumor. Thus, patients expressing ALDH1 often found associated with greater depth of invasion, poor differentiation and lymph node metastasis (Chen et al., 2015).

In gastric carcinoma, expression of ALDH1 was found to be significantly associated with invasion, stage and lymph node metastasis. Additionally, the survival analysis done by Kaplan-Meier method reported that the patients over expressing ALDH1 have significantly shorter OS and recurrence-free survival (RFS) than the patients with low expression of ALDH1. In gastric carcinoma, ALDH1 also appears to be as a potential prognostic marker which plays a key role in tumor progression (Li et al., 2014).

In HNSCC till now, ALDH1 has been considered as one of the discrete markers of CSCs which plays a pivotal role in the maintenance of properties of CSCs (Clay et al. 2010; Chen et al., 2011). Numerous studies were performed with the cell lines of HNSCC documented that ALDH1+ cells were tumorigenic and exhibits chemo and radio-resistance (Singh and Settleman 2010; Chen et al., 2011). These findings accentuate ALDH1 as a potential marker for prognosis of HNSCC as well as an appropriate target for therapeutic success (Qian et al., 2014).

In current study, the results were corroborated with the previous studies. The current study was based on an Indian population which demonstrates that HNSCC patients expressing ALDH1 stimulate tumor invasion, progression, recurrence and treatment failure. Our results showed that not only ALDH1 but other TNM factors such as lymph node status and grade could also be considered as a potential marker for tumor progression, therapy resistance and as an independent predictive marker for OS. Ang et al., (2010) have indicated a clear association between smoking habits and OS in North American patient population. Taking this into account, in current study, we do not find any correlation with tobacco. On the contrary, alcohol was found to be an independent predictive marker of radiotherapy along with ALDH1.

In advanced stage HNSCC, patients were subjected to radiotherapy or chemo-radiotherapy. However, usually patients exhibit local lymph node metastasis or distant metastasis as well and recurrence because of radiation resistance of tumor cells which concludes as a failure of radiotherapy treatment. In this study, we evaluate the predictive significance of ALDH1 in terms of radiotherapy resistance and OS. On evaluating the expression of ALDH1, we found that over expression of ALDH1 results into the elevated resistance to the radiotherapy. In other words, inhibition of ALDH1 could increase the radiotherapy sensitivity in advanced stage HNSCC. Though, an in-depth research is well warranted to discover the role or underlying mechanism of ALDH1 which leads to radioresistance. In conclusion, the presence of CSCs within tumors is known for restoring the tumor during and after standard chemo-radiotherapy. ALDH1 is a putative marker for CSCs. In this study, it is found to be over expressed. Advanced stage HNSCC patients with over expression of ALDH1 did not respond to standard radiotherapy treatment. In addition, study also found that patients with high ALDH1 expression, positive lymph node status, and high grade showed poor overall survival. Therefore, they can serve as predictive markers for treatment and survival. However, further investigations are required to unravel the mechanism of ALDH1 in treatment failure and recurrence.

\section{Author Contribution Statement}

Conception and design: MadanLal Brahma Bhatt and Parul Dubey. Analysis and interpretation of data: MadanLal Brahma Bhatt, Parul Dubey and Smrati Bhadauria. Drafting, review and/or revision of the manuscript: MadanLal Brahma Bhatt, Parul Dubey, Smrati Bhadauria, Rajeev Gupta, Anupam Mishra, Vijay Kumar and Narayan Kumar. Final approval: MadanLal Brahma Bhatt and Smrati Bhadauria

\section{Acknowledgements}

\section{Funding statement}

This study was financially supported by the Institutional/In-house funds from King George's Medical University, Lucknow, India.

This project is a part of an approved student thesis.

\section{Disclosure of Potential Conflicts of interest}

The authors declare no conflict of interest.

\section{Ethical Approval Statement}

The Institutional Ethics Committee has approved the study protocol, which follows the Declaration of Helsinki. The written informed consent has been obtained from all the patients included in this study.

\section{Availability of Data}

The data that support the findings of this study are available on request from the corresponding author. The data are not publicly available due to privacy or ethical restrictions.

\section{References}

Alamgeer M, Ganju V, Szczepny A, et al (2013). The prognostic significance of aldehyde dehydrogenase 1A1 (ALDH1A1) and CD133 expression in early stage non-small cell lung cancer. Thorax, $\mathbf{0}, 1-0$.

Alamgeer M, Peacock CD, Matsui W, Ganju V, Watkins DN (2013). Cancer stem cells in lung cancer: Evidence and controversies. Respirology, 18, 757-4.

Alison MR, Lin WR, Lim SM, Nicholson LJ (2012). Cancer stem cells: in the line of fire. Cancer Treat Rev, 38, 589-8.

Al-Hajj M, Wicha MS, Benito-Hernandez A, et al (2003). Prospective identification of tumorigenic breast cancer cells. Proc Natl Acad Sci U S A, 100, 3983-8.

Ang KK, Harris J, Wheeler R g et al (2010). Human papillomavirus and survival of patients with oropharyngeal cancer. $N$ Engl 
JMed, 363, 24-5.

Baumann M, Krause M, Hill R (2008). Exploring the role of cancer stem cells in radioresistance. Nat Rev Cancer, $\mathbf{8}$, $545-4$.

Black WJ, Stagos D, Marchitti SA, et al (2009). Human aldehyde dehydrogenase genes: alternatively spliced transcriptional variants and their suggested nomenclature. Pharmacogenet Genomics, 19, 893-2.

Chan KS, Espinosa I, Chao M, et al (2009). Identification, molecular characterization, clinical prognosis, and therapeutic targeting of human bladder tumor-initiating cells. Proc Natl Acad Sci U S A, 106, 14016-1.

Chen C, Wei Y, Hummel M, et al (2011). Evidence for epithelialmesenchymal transition in cancer stem cells of head and neck squamous cell carcinoma. PLoS One, 6, e16466.

Clarke DW, Palle K (2016). Aldehyde dehydrogenases in cancer stem cells: potential as therapeutic targets. Ann Transl Med, 4, 518 .

Clay MR, Tabor M, Owen JH, et al (2010). Single-marker identification of head and neck squamous cell carcinoma cancer stem cells with aldehyde dehydrogenase. Head Neck, 32, 1195-1.

Collina F, Bonito MD, Bergolis VL, et al (2015). Prognostic value of cancer stem cells markers in triple-negative breast cancer. Biomed Res Int, 2015, 158682.

Economopoulou P, de Bree R, Kotsantis I, Psyrri A (2019). Diagnostic tumor markers in head and neck squamous cell carcinoma (HNSCC) in the clinical setting. Front Oncol, 9, 827.

Eisenhauer EA, Therasse P, Bogaerts J, et al (2009). New response evaluation criteria in solid tumours: Revised RECIST guideline (version 1.1). Eur J Cancer, 45, 228-7.

Fitzmaurice C, Allen C, Barber RM, et al (2017). Global, regional, and national cancer incidence, mortality, years of life lost, years lived with disability, and disability-adjusted life-years for 32 cancer groups, 1990 to 2015: a systematic analysis for the global burden of disease study. JAMA Oncol, 3, 524-8.

Jordan CT, Guzman ML, Noble M (2006). Cancer stem cell. $N$ Engl J Med, 355, 1253-1.

Kim CF, Jackson EL, Woolfenden AE, et al (2005). Identification of bronchialveolar stem cells in normal lung and lung cancer. Cell, 121, 823-5.

Lapidot T, Sirard C, Vormoor J, et al (1994). A cell initiating human acute myeloid leukaemia after transplantation into SCID mice. Nature, 367, 645-8.

Liu Y, Lv DL, Duan JJ, et al (2014). ALDH1A1 expression correlates with clinicopathologic features and poor prognosis of breast cancer patients: a systematic review and metaanalysis. BMC Cancer, 14, 444.

Li XS, Xu Q, Fu XY, Luo WS (2014). ALDH1A1 overexpression is associated with the progression and prognosis in gastric cancer. BMC Cancer, 14, 705.

Mannelli G, Gallo O (2012). Cancer stem cells hypothesis and stem cells ion head and neck cancers. Cancer Treat Rev, 38, 515-9.

Martin M, Hinojar A, Cerezo L et al (2016). Aldehyde dehydrogenase isoform 1 (ALDH1) expression as a predictor of radiosensitivity in laryngeal cancer. Clin Transl Oncol, 18, 825-0.

Ma SR, Wang WM, Huang CF, Zhang WF, Sun ZJ (2015). Anterior gradient protein 2 expression in high grade head and neck squamous cell carcinoma correlated with cancer stem cell and epithelial mesenchymal transition. Oncotarget, 6, 8807-1.

McCullough MJ, Prasad G, Farah CS (2010). Oral mucosal malignancy and potentially malignant lesions: an update on the epidemiology, risk factors, diagnosis and management. Australian Dent J, 55, 61-5.

Patel SG, Shah JP (2005). TNM staging of cancers of the head and neck: striving for uniformity among diversity. $C A$ Cancer J Clin, 55, 242-8.

Prince ME, Sivanandan R, Kaczorowski A, et al (2007). Identification of a subpopulation of cells with cancer stem cell properties in head and neck squamous cell carcinoma. Proc Natl Acad Sci U S A, 104, 973-8.

Qian Xu, Wagner S, Ma C et al (2014). Prognostic significance of ALDH1A1-positive cancer stem cells in patients with locally advanced, metastasized head and neck squamous cell carcinoma. J Cancer Res Clin Oncol, 140, 1151-8.

Ricci-Vitiani L, Lombardi DG, Pilozzi E, et al (2007). Identification and expansion of human colon-cancerinitiating cells. Nature, 445, 111-5.

Shaw R, Beasley N (2016). Aetiology and risk factors for head and neck cancer: United Kingdom National Multidisciplinary Guidelines. J Laryngol Otol, 130, $2-9$.

Shen JX, Liu J, Li GW, Huang YT, Wu HT (2016). Mining distinct aldehyde dehydrogenase 1 (ALDH1) isoenzymes in gastric cancer. Oncotarget, 7, 25340-9.

Siegel RL, Miller KD, Jemal A (2017). Cancer statistics, 2017. CA Cancer J Clin, $67,0-7$.

Singh SK, Clarke ID, Terasaki M, et al (2003). Identification of a cancer stem cell in human brain tumors. Cancer Res, 63, 5821-8.

Singh S, Brocker C, Koppaka V, et al (2013). Aldehyde dehydrogenases in cellular responses to oxidative/ electrophilic stress. Free Radic Biol Med, 56, 89-1.

Singh S, Arcaroli J, Thompson DC, Messersmith W, Vasiliou V (2015). Acetaldehyde and Retinaldehyde Metabolizing Enzymes in Colon and Pancreatic Cancers. In Biological Basis of Alcohol-Induced Cancer, 815, pp 281-4.

Singh A, Settleman J (2010). EMT, cancer stem cells and drug resistance: an emerging axis of evil in the war on cancer. Oncogene, 29, 4741-1.

Weissman IL, Anderson DJ, Gage F (2001). Stem and progenitor cells: Origins, phenotypes, lineage commitments, and transdifferentiations. Annu Rev Cell Dev Biol, 17, 387-3.

Wu PC, Lai VC, Fang JW, et al (1999). Hepatocellular carcinoma expressing both hepatocellular and biliary markers also expresses cytokeratin 14, a marker of bipotential progenitor cells. J Hepatol, 31, 965-6.

Wu S, Xue W, Huang X, et al (2015). Distinct prognostic values of ALDH1 isoenzymes in breast cancer. Tumor Biol, 36, 2421-6.

You Q, Guo H, Xu D (2014). Distinct prognostic values and potential drug targets of ALDH1 isoenzymes in non-smallcell lung cancer. Drug Design Development Ther, 9, 5087-7.

Zhou C, Sun B (2014). The prognostic role of the cancer stem cell marker aldehyde dehydrogenase 1 in head and neck squamous cell carcinomas: A meta-analysis. Oral Oncol, 50, 1144-8.

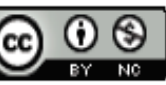

This work is licensed under a Creative Commons AttributionNon Commercial 4.0 International License. 